\title{
A novel mouse model of nonalcoholic steatohepatitis with significant insulin resistance
}

\author{
Yuriko Adkins ${ }^{1,2}$, Iwan W Schie ${ }^{3}$, Dawn Fedor ${ }^{2}$, Aurosis Reddy ${ }^{1}$, Samantha Nguyen ${ }^{4}$, Ping Zhou ${ }^{5}$, \\ Darshan S Kelley ${ }^{1,2}$ and Jian $\mathrm{Wu}^{4,5,6}$
}

Currently available models insufficiently reflect the pathogenic alternation of nonalcoholic steatohepatitis $\backslash$ NASH), such as insulin resistance. The present study aimed to characterize a novel NASH model caused by feeding the diet containing conjugated linoleic acid (CLA). In this study, mice were fed a control diet or the diet containing 0.5\% CLA for 8 weeks. The insulin tolerance test (ITT) and homeostasis model assessment of insulin resistance (HOMA-IR) were used to determine the extent of insulin resistance. Liver lipotoxicity and inflammation were assessed by endoplasmic reticulum (ER) stress, autolipophagy, recruitment of Kupffer cells and hepatic stellate cell (HSC) activation. We found that liver weight was markedly increased, and histopathological examination showed marked macrosteatosis with focal hepatocellular death through apoptosis, and mild pericellular fibrosis with Kupffer cell recruitment and HSC activation, as well as light chain III $\beta$-positive cells and enhanced ER stress in mice fed the CLA-containing diet. Enhanced synthesis and reduced $\beta$-oxidation of fatty acids resulted in their accumulation and lipotoxicity in hepatocytes. A biophotonic technology revealed lipid droplet accumulation in the liver from mice fed the CLA-containing diet, and Raman spectroscopic analysis indicated that these lipid droplets predominantly contained saturated fatty acids. Elevated fasting insulin levels, abnormal ITT and HOMA-IR confirmed the marked insulin resistance in these mice. Decreased phosphorylation of the insulin-signaling molecule Akt was partially responsible for the significant insulin resistance. In conclusion, Mice fed the diet containing CLA-developed steatohepatitis with marked insulin resistance, which is similar to the characteristics observed in NASH patients. The further characterization of this model would be particularly useful for revealing the critical role of insulin resistance in NASH development in conditions such as metabolic syndrome, diabetes and obesity. Laboratory Investigation (2013) 93, 1313-1322; doi:10.1038/labinvest.2013.123; published online 21 October 2013

KEYWORDS: animal model; insulin resistance; lipid droplets; nonalcoholic steatohepatitis; raman micro-spectroscopy

Over $35 \%$ of the US population is obese, and the resulting insulin resistance leads to a high prevalence of metabolicassociated disorders, such as diabetes, nonalcoholic fatty liver disease (NAFLD), metabolic syndrome, hyperlipidemia, hypertension and vascular abnormalities in various organs. ${ }^{1}$ NAFLD has become a major factor in abnormal liver biochemical tests. Nonalcoholic steatohepatitis (NASH) is one stage of NAFLD, and significantly contributes to endstage liver disease (ESLD) and the increasing incidence of hepatocellular carcinoma. ${ }^{2,3}$ Nearly $20 \%$ of NASH patients will progress to cirrhosis with various complications. When
NASH is diagnosed, 5- and 10-year survival rates are 67 and $59 \%$, respectively, ${ }^{4}$ and it is becoming the main disorder requiring a liver transplantation. ${ }^{1}$ In many cases, NASH is the hepatic manifestation of a systemic disorder, such as type II diabetes, metabolic syndrome or hyperlipidemia, in which insulin resistance is a common feature. Insulin sensitizers, such as biguanides and thiazolidinediones are used for NASH treatment in clinical studies. ${ }^{2}$ Pioglitazone has been shown to significantly improve steatosis and inflammation, but not hepatic fibrosis or progression to ESLD in NASH patients. ${ }^{3}$ Antioxidant vitamin $\mathrm{E}$ has been shown to be beneficial for

\footnotetext{
${ }^{1}$ Western Human Nutrition Research Center, Agricultural Research Service, USDA, Davis, CA, USA; ${ }^{2}$ Department of Nutrition, University of California, Davis, Davis, CA, USA; ${ }^{3}$ Center for Biophotonics Sciences and Technology, University of California, Davis Medical Center, Sacramento, CA, USA; ${ }^{4}$ Division of Gastroenterology and Hepatology, Department of Internal Medicine, University of California, Davis Medical Center, Sacramento, CA, USA; ${ }^{5}$ Stem Cell Program, University of California, Davis Medical Center, Sacramento, CA, USA and ${ }^{6}$ Key Laboratory of Molecular Virology, Fudan University Shanghai Medical College, Shanghai, China

Correspondence: DS Kelley, PhD, Western Human Nutrition Research Center, Agricultural Research Service, USDA, Davis 430 West Health Sciences Drive, Davis, CA 95616, USA or Professor J Wu, MD, PhD Division of Gastroenterology and Hepatology, Department of Internal Medicine, UC Davis Medical Center, Institute for Regenerative Cures, Suite 1610, 2921 Stockton Boulevard, Sacramento, CA 95817, USA.

E-mail: Darshan.Kelley@ars.usda.gov or jdwu@ucdavis.edu

Received 15 July 2013; accepted 12 September 2013
} 
selected patients, and it is recommended in the AASLD practice guideline. ${ }^{3,4}$ Other nutritional supplements, such as n-3-polyunsaturated fatty acid (PUFA), ${ }^{5}$ pentoxifylline, ${ }^{6}$ coffee $^{7}$ and coumarin ${ }^{8}$ have been investigated in animal models of NASH to determine their benefits in decreasing fatty accumulation, lipotoxicity and progression to fibrosis/ cirrhosis.

There are a number of NASH animal models available; the most common ones are mice fed the methionine/cholinedeficient diet (MCD) or a high fat/high-calorie diet (HFC). ${ }^{9}$ The MCD diet causes hepatic steatosis with loss of body weight, and no insulin resistance occurs in these mice. The HFC diet causes obesity, hepatic steatosis with mild injury and insulin resistance. Genetic deficient ob/ob (leptin deficient) or $\mathrm{db} / \mathrm{db}$ mice (defect in leptin receptor) ${ }^{9}$ or Zucker rats ${ }^{10}$ do not develop steatosis automatically, and they often need to be fed either MCD or HFC diet. STAM mice, which develop steatohepatitis, cirrhosis and carcinoma in progression starting treatment with streptozotocin at day 2 and feeding the HFC diet at 4 weeks, develop NASH at 8 weeks and cancer at 16 weeks. ${ }^{11}$ However, in these models no significant insulin resistance develops, which insufficiently reflects the pathogenesis of NASH in patients as a component/complication of obesity, diabetes and metabolic syndrome.

Trans fatty acids (TFA) are considered unhealthy, because they increase an n-6 to n-3 PUFA ratio in tissues. One TFA in the diet is conjugated linoleic acid (CLA), which refers to a mixture of positional and geometric isomers of linoleic acid $(18: 2$; n6). One isomer, trans-10, cis-12 CLA, is found in partially hydrogenated vegetable oil and in processed foods. CLA has been claimed to reduce depot fat and increase muscle mass in humans, which has led to a booming trade in over-the-counter sale of a mixture of CLA isomers. Other combinations of CLA isomers have caused fatty liver and insulin resistance in human subjects. We have previously reported that mice fed a diet containing CLA developed severe fat accumulation in the liver. ${ }^{12}$ Here, we further characterize this model, and our findings demonstrate that mice fed the CLA-containing diet developed typical NASH in histopathology with a characteristic insulin resistance, in addition to marked oxidant stress and lipotoxicity, and that the insulin resistance has a critical role in the initiation and progression of NASH towards fibrosis.

\section{MATERIALS AND METHODS}

\section{Experimental Animals and Diets}

The animal protocol was approved by the Institutional Committee for the Animal Use and Care (IACUC) of University of California, Davis, CA, USA. Eight-week-old, pathogen-free C57BL/6N female mice (Charles River, Raleigh, NC, USA) were maintained at an animal facility of the Center for Laboratory Animal Care and Science (CLAS) at University of California, Davis. All experiments were in compliance with the guidelines of the National Institutes of
Health for experimental animals. The room temperature was maintained at $25^{\circ} \mathrm{C}$ with a 12 -h dark/light cycle. All mice were fed a regular chow diet for 1 week before they were switched to a modified AIN93G diet (soybean oil replaced with corn oil), which was used as the control diet. Trans-10, cis- 12 conjugate linoleic acid (CLA) at $0.5 \%$ (90\% purity; Larodan Fine Chemicals, Malmö, Sweden) of total food weight was added to form the CLA-containing diet. The other components of the control and CLA diets were similar except that the control diet contained 5\% corn oil, whereas the CLA-diet contained $0.5 \%$ CLA plus $4.5 \%$ corn oil. Each group had 6 mice. Fresh diets were served daily, and the food left from the previous day was weighed and recorded.

\section{Insulin Tolerance Test}

After 7 weeks of feeding, mice were fasted for $4 \mathrm{~h}$ without the restriction of water, and were injected intraperitoneally with insulin $(0.55 \mathrm{mU} / \mathrm{kg}$ body weight $)$ diluted with normal saline. Blood was obtained from the saphenous vein $0,15,30,60,90$ and $120 \mathrm{~min}$ after insulin injection. Plasma glucose was measured with a portable glucometer (One Touch Ultra 2, Lifescan, Milpitas, CA, USA). Homeostasis model assessment of insulin resistance (HOMA-IR) was calculated based on fasting insulin and glucose levels before killing according to the methods we reported previously. ${ }^{13}$

\section{Animal Killing, Tissue Collection and Immunohistochemistry}

All mice were terminated 8 weeks after feeding with either the control diet or CLA-containing diet by $\mathrm{CO}_{2}$ inhalation after overnight fasting. Blood was collected by heart puncture and serum insulin was measured by an ELISA kit using mouse insulin as a standard (Crystal Chem Inc., Downers Grove, IL, USA). Liver specimens were collected, weighed, frozen in liquid nitrogen and then stored at $-80{ }^{\circ} \mathrm{C}$ for future analysis. A portion of liver tissue was fixed in $10 \%$ neutralized formalin, embedded in paraffin and sectioned in $4 \mu \mathrm{m}$ thickness. Sections from paraffin-embedded tissue were stained with hematoxylin and eosin (H\&E) or trichrome staining for fibrotic deposition. ${ }^{14}$ After deparaffinization, apoptotic cells in liver tissue were stained with an in situ terminal deoxynucleotidyl transferase-mediated dUTP nick end labeling (TUNEL) assay as previously described. ${ }^{15}$ Activated hepatic stellate cells (HSCs) were visualized by staining of smooth muscle $\alpha$-actin (SMA) as we described previously. ${ }^{16}$ Kupffer cells were identified by staining with polyclonal goat antibodies against CD163 (from Santa Cruz Biotechnologies, Santa Cruz, CA, USA) as described previously by us. ${ }^{17}$ Microtubule-associated proteins light chain III- $\beta$ (LCIII $\beta$ ) was stained in liver sections using polyclonal rabbit antibody from Sigma Chemical Co (St Louis, MO, USA) as described previously. ${ }^{18}$ All micrograph images were taken with a Keyence fluorescence microscope. 
Imaging of Lipid Droplets in Mouse Liver Using Coherent Anti-Stokes Raman Scattering (CARS) Microscopy and Analysis of Lipid Content With Raman Micro-Spectroscopy

Frozen liver tissues at $60 \mu \mathrm{m}$ were sectioned for CARS imaging without fixation. CARS images of the frozen liver tissue sections were acquired on a noncommercial CARS microscope as described previously. ${ }^{19}$ Briefly, the CARS signal was generated by combining a $1064 \mathrm{~nm}$, short-pulsed diodepumped Nd:YVO , laser (PicoTrain, HighQ Laser, Austria), with an $817 \mathrm{~nm}$ Stokes-excitation from an optical parametric oscillator (OPO Levante, APE, Germany). This excitationwavelength combination drives coherently the $2845 \mathrm{~cm}^{-1}$ $\mathrm{CH}_{2}$-stretch vibration of the aliphatic chain of fatty acids. The generated CARS signal was separated by a combination of a set of longpass filters (Semrock, Rochester), and after passing a spectrograph (Acton SpectraPro 2300i, PI Acton), the signal was detected on an avalanche photo diode (APD, Perkin-Elmer). Raman spectra were subsequently acquired from the cellular lipid droplets in liver tissue sections. The Raman signal was excited by a $785-\mathrm{nm}, \mathrm{cw}$-laser (CrystaLaser), and after passing a spectrometer detected on a back-illuminated deep-depletion CCD camera (PIXIS 100BR, PI Action). The raw Raman spectra were background corrected, based on a modified Lieber method and area-normalized.

\section{Quantitative RT-PCR for Relative Gene Expression}

Total RNA was isolated from the liver tissue using the PureLink Micro-to-Midi purification kit according to manufacturer's instructions (Invitrogen, Carlsbad, CA, USA). RNA quality and integrity was determined using an Agilent 2100 Bioanalyzer (Agilent Technologies, Palo Alto, CA, USA). RNA abundance for the CCAAT/enhancer-binding homologous protein $(\mathrm{CHOP})$, carnitine palmitoyl transferase- $1 \alpha(\mathrm{CPT} \alpha 1)$, peroxisome proliferator-activated receptor- $\alpha(\operatorname{PPAR} \alpha)$, sterol regulatory element-binding protein-1 (SREBP1), acetyl CoA carboxylase (ACC) and fatty acid synthesis (FAS), acyl-CoA oxidase 1 (ACOX1), stearoyl-CoA desaturase (SCD1) was determined by using gene-specific Taqman primers and FAMMGB-labeled probes (Applied Biosystems, Foster City, CA, USA). Real-time RT-PCR reactions were conducted on a 384well plate in triplicates and was carried out using ABI 7900HT Fast Real-Time PCR System and documented by SDS software v.2.2.2 (Applied Biosystems). Relative gene expression was calculated using the $2^{-\Delta \Delta \mathrm{Ct}}$ method, which normalizes against the mouse $\beta$-actin gene and the control group computed average $\Delta \mathrm{Ct}^{20}$

\section{Protein Extraction and Western Blot Analysis}

Frozen liver samples were homogenized in ice-cold T-Per tissue protein extraction reagent with Halt protease and phosphatase inhibitor cocktail (Pierce/Thermo Fisher, Rockford, IL, USA) according to manufacturer's instructions. Lysate supernatants were collected by centrifugation and total protein concentration was determined with the Bradford assay. Equal volume and quantity of the supernatants were resolved on a $10 \%$ SDS-PAGE gel followed by transfer of the proteins onto a polyvinylidene difluoride membrane (Bio-Rad, Hercules, CA). A molecular weight marker (BioRad) was run concurrently for protein identification. The membrane was blocked in 5\% bovine serum albumin in TBST buffer (20 mM Tris (pH 7.5), $500 \mathrm{mM} \mathrm{NaCl}$ and $0.1 \%$ (v/v) Tween 20) and probed with specific antibodies against pAkt $\left(\mathrm{Thr}^{308}\right.$ ), total Akt and GAPDH (Cell Signaling Technology, Danvers, MA, USA) at $4{ }^{\circ} \mathrm{C}$ overnight followed by incubation with the appropriate horseradish peroxidaseconjugated secondary antibody. Immunoreactive bands were visualized using an enhanced chemiluminescence reaction (WesternBright Quantum, Advansta, Menlo Park, CA, USA). Blots were imaged for $60 \mathrm{~s}$ to $2 \mathrm{~min}$ on a ChemiDoc XRS + Imager and quantified using the associated Image Lab software (Bio-Rad).

\section{Statistical Analysis}

The quantitative data were expressed as means \pm s.e.m. and the data comparing control diet group and CLA-containing diet group were analyzed by unpaired Student's $t$-tests. A $P$-value of $<0.05$ was considered as statistically significant.

\section{RESULTS}

\section{Liver Weight and Histopathology}

After 8 weeks of feeding, the mean liver weight in the CLA diet group was 50\% more than that in the control diet group ( $1.5 \pm 0.1$ vs $1.0 \pm 0.0 \mathrm{~g}, P<0.01)$. The mice receiving the control diet displayed mild histological changes in their livers, manifests as enlarged hepatocytes with mild micro lipid droplets and no focal cell death was observed (Figure 1a). However, the livers from mice receiving the CLA-diet exhibited typical macrosteatosis and focal cell death (Figure $1 \mathrm{~b}$ and $\mathrm{c}$ ). The inflammatory infiltration was observed in the portal zone and the area with cell death. It appears that more apoptotic cells were observed in the representative areas of the livers in the CLA-diet group than the control mice (Figures $1 \mathrm{~d}$ and e).

\section{Fibrotic Deposition in the Liver}

Mouse liver was stained with Masson's Trichrome staining to reflect the connective tissue deposition. As shown in Figure 2a, very little positive staining was found in the portal zone and around the central vein area in the livers of the control mice. In contrast, the livers from the CLA-diet group displayed thin septa formation stretching from the portal zone to the parenchyma of the lobule. There was significant pericellular fibrotic deposition, which is typically seen in the human NASH liver (Figure 2b). Consistent with the fibrotic deposition, more activated HSCs, as reflected by positive smooth muscle $\alpha$-actin (SMA) staining, were seen in the sinusoids and the portal zone area in the livers of the CLAdiet group compared with those in the control diet group (Figures $2 \mathrm{c}$ and $\mathrm{d}$ ). 

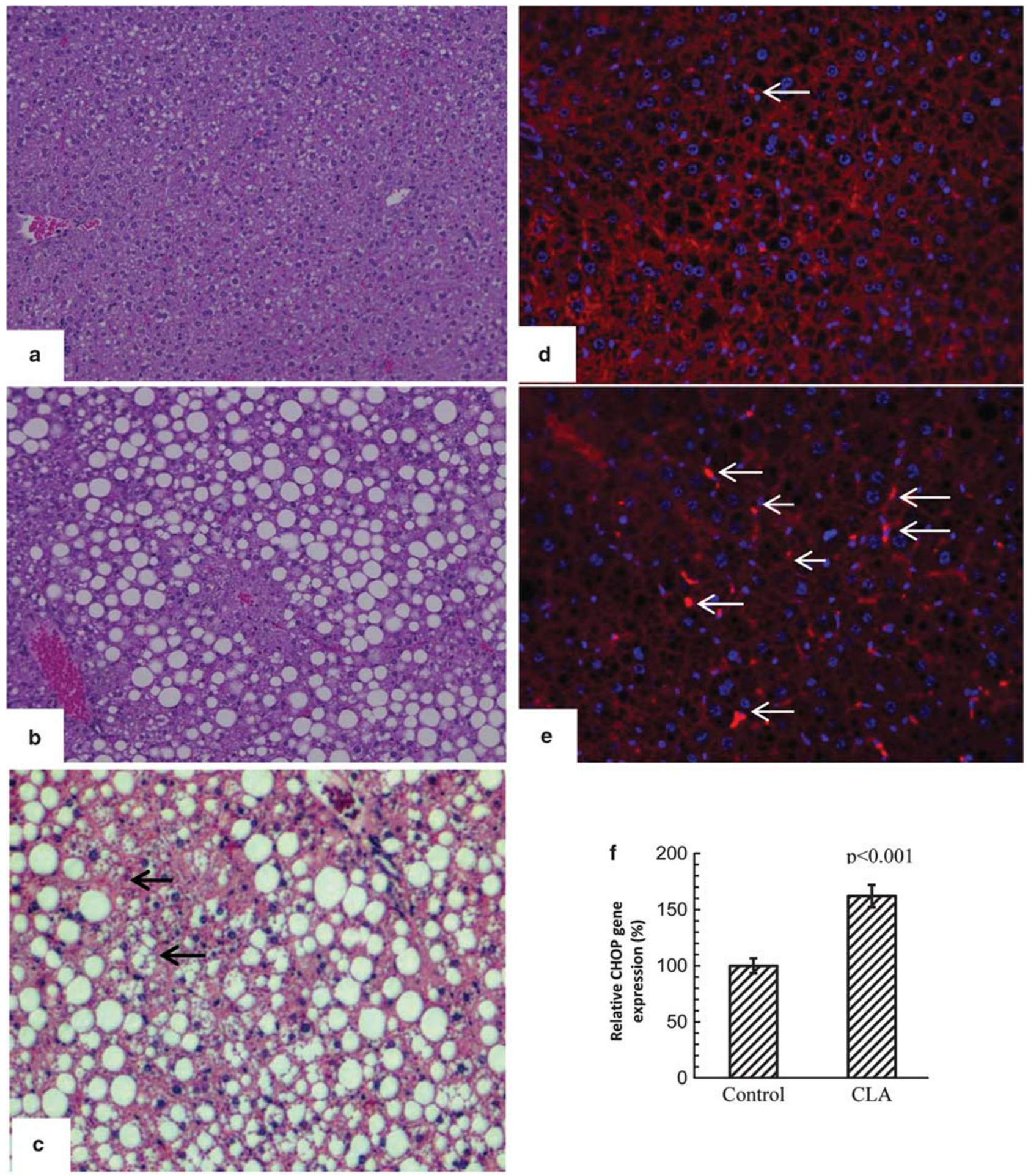

Figure 1 Liver histology of mice fed the conjugated linoleic acid (CLA)-containing diet for 8 weeks. Representative micrographs of liver histology with hematoxylin and eosin (H\&E) staining from mice fed the control diet (a) and the CLA-containing diet (b) $(\times 200)$. Focal-cell death in the liver from the CLA-containing diet group is indicated in panel c. In situ TUNEL (terminal deoxynucleotidyl transferase-mediated dUTP nick end labeling) staining of apoptotic cells was visualized in red $(\mathbf{d}, \mathbf{e})$ and indicated with arrows $(\times 200)$. (f) CHOP mRNA levels determined by quantitative RT-PCR $(n=6)$.

\section{Enhanced Recruitment of Kupffer Cells}

In order to determine whether Kupffer cells are involved in lipotoxicity and inflammatory process, CD163 staining was used to identify Kupffer cell recruitment in the mouse liver. As shown in Figure 2, few CD163-positive cells were visualized in the representative liver of the control diet group 

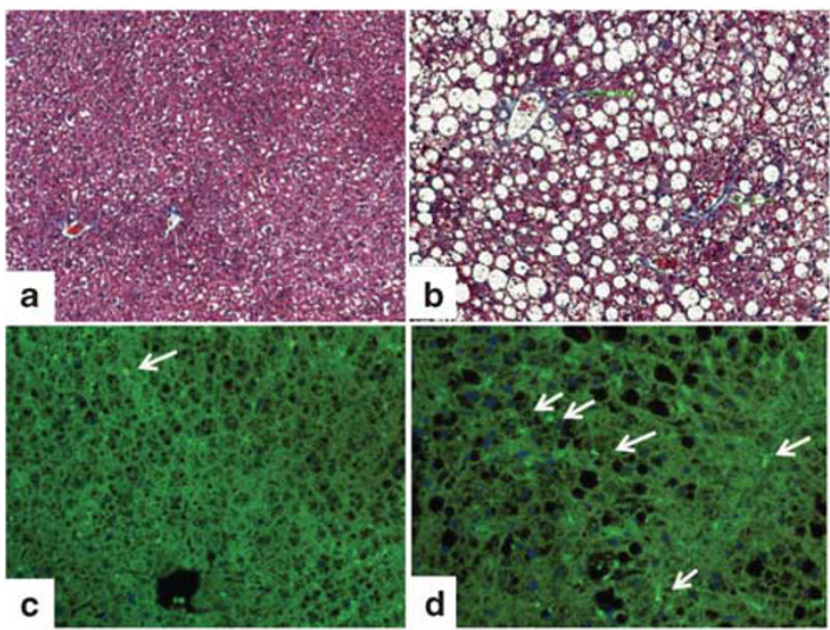
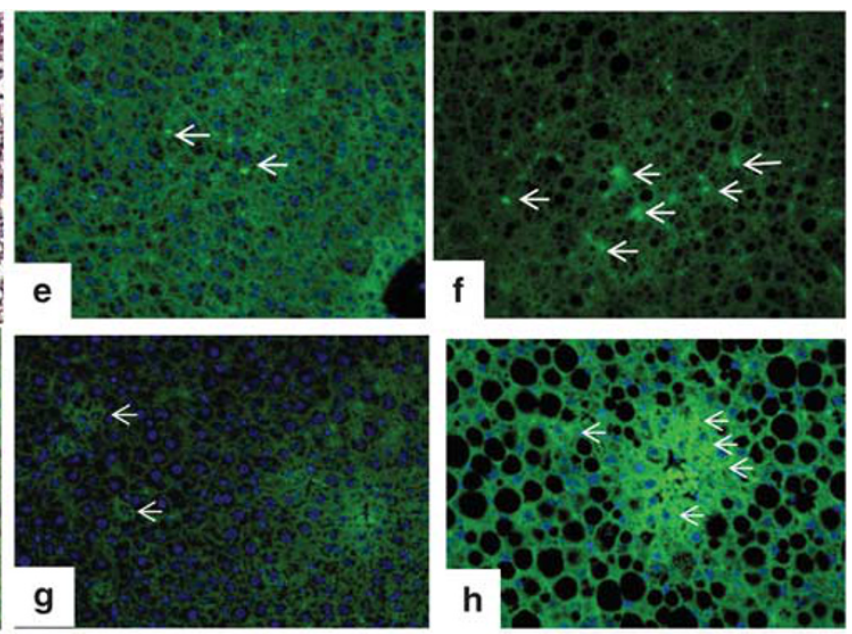

Figure 2 Hepatic fibrosis and activation of hepatic stellate cells (HSCs), Kupffer cell recruitment and markers of autophagy. Masson's Trichrome staining was performed for deposition of connective tissue in the mouse liver, and visualized in blue. Representative micrographs (a, b) are from the control diet group and conjugated linoleic acid (CLA)-containing diet group ( $\times 100)$. Activated HSCs in mouse liver from the control diet (c) and CLA-

containing diet (d) groups were stained by antibodies against SMA, and visualized in green. The sections were count-stained with 4',6-diamidino-2phenylindole (DAPI) for nuclei $(\times 400)$. Kupffer cells are visible after immunohistochemical staining with monoclonal antibodies against CD163 in the liver of CLA-diet-fed mice (f) than the controls (e), and DAPI counter-staining for nuclear visualization ( $\times 400)$. More LCIII $\beta$-positive cells are visible in the liver of mice fed the CLA-containing diet for 8 weeks $(\mathbf{h})(\times 400)$ compared with those fed the control diet $(\mathbf{g})$.

(Figure 2e). However, it appears that many more CD163positive cells were seen in the sinusoidal areas in the mice from the CLA-diet group (Figure 2f), indicating that there was enhanced recruitment from the bloodstream or activation of the resident macrophages in the mice fed the CLAcontaining diet.

\section{Increased Autophagy}

Microtubule-associated proteins light chain III- $\beta$ (LCIII $\beta$ ) is considered to be a reliable marker for autophagy, a process for a cell to save energy during starvation by engulfing itself. Microlipophagy is thought to be an important process for a cell with fat accumulation to clear excess lipid. Thus, staining of LCIII $\beta$ in fatty liver may reflect the clearance of lipid droplets. As shown in Figure $2 \mathrm{~g}$, few LCIII $\beta$-positive cells were seen in a representative liver from the control diet group, whereas many more LCIII $\beta$-positive cells were found in the central vein area in the liver from the CLA-diet group (Figure 2h), indicating that there was a much accelerated lipid clearance in hepatocytes with a striking lipid droplet formation.

\section{Enhanced Endoplasmic Reticulum (ER) Stress in Steatotic liver}

ER stress may lead to insulin resistance and apoptosis, and may also affect autophagy. We determined CHOP mRNA levels by quantitative RT-PCR, and the data are shown in Figure 1f. It was found that $\mathrm{CHOP}$ gene expression was strikingly upregulated in the liver of mice fed the CLAcontaining diet, indicating that there was enhanced ER stress due to lipid accumulation, oxidant stress and lipotoxicity in hepatocytes.

\section{Biophotonic Technologies for Visualization of Lipid Droplets in Mouse Liver}

CARS was employed to visualize lipid droplets in liver tissue and Raman spectroscopy to determine the molecular composition of lipid droplets in liver sections. In order to avoid the clearance of lipid droplets by a fixative, we used unfixed liver sections for imaging acquisition (Figures $3 a$ and b). Lipid droplets were clearly visible in the frozen liver sections from mice fed the CLA-containing diet (Figure 3b). Only a few small intracellular lipid droplets were visualized in the liver tissue of mice fed the control diet. These observations confirmed our observation from transmission microscopy as shown in Figures $1 \mathrm{~b}$ and $\mathrm{c}$.

By using Raman spectroscopy, we were able to further determine the molecular composition and the fatty acid profile in the visualized lipid droplets. A mean Raman spectrum of 20 lipid droplets from the livers of mice fed the CLA-containing diet is shown in Figure 3c. The peak location and intensity indicate the molecular bonds present in the samples and the abundance of the molecular bond in the sections, respectively. The ratio of relative intensity of the saturated peak due to a $\mathrm{CH} 2$ vibration at $1444 \mathrm{per} \mathrm{cm}$ to that of the unsaturated peak due to a $\mathrm{C}=\mathrm{C}$ stretch vibration at 1660 per $\mathrm{cm}$ indicates that the majority of the fatty acids in the lipid droplets were saturated. Moreover, the presence of the peak at 1730 per $\mathrm{cm}$ implies that the fatty acids in the lipid droplets were in an esterified form.

\section{Insulin-Signaling Defect Partially Responsible for Insulin Resistance}

In order to determine the extent of insulin resistance, we performed an insulin tolerance test (ITT) in both groups. 
There was no significant difference in the basal glucose levels between these two groups (Figure 4a). After injection of insulin, the serum glucose levels dropped in both groups, but
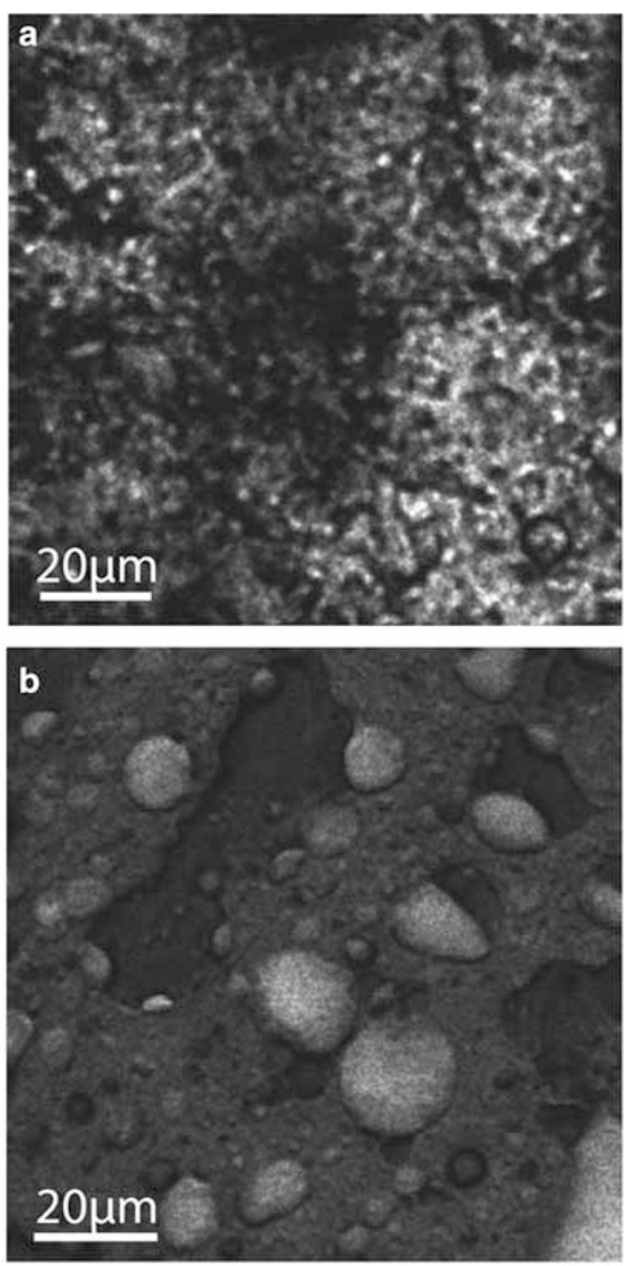

c

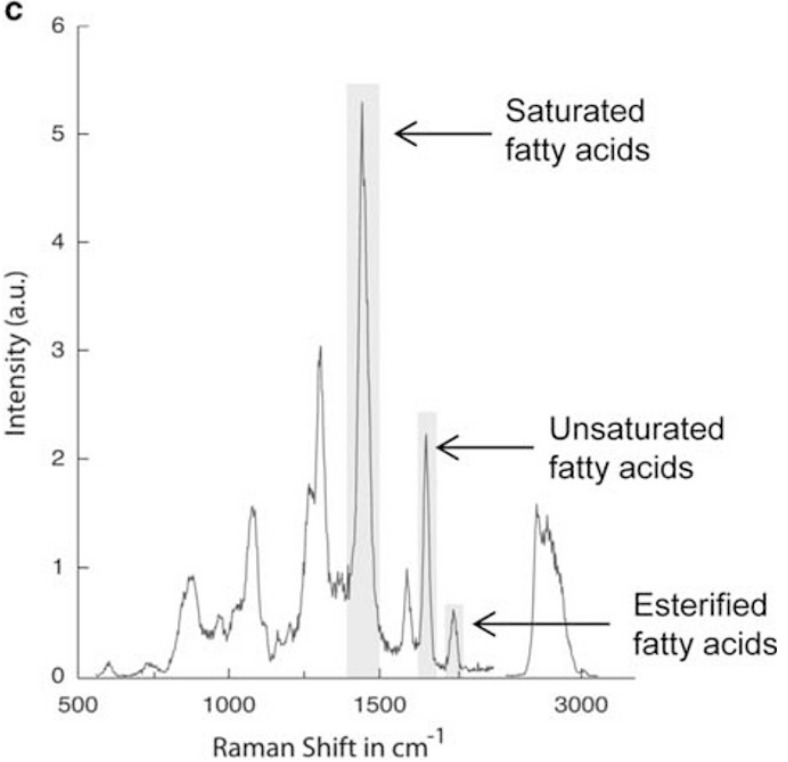

the decrease $(23.8 \%)$ in glucose level in mice fed with CLAcontaining diet was less than that in the control diet group $(41.5 \%, P<0.01)$ during the first $15 \mathrm{~min}$. After that, glucose levels rebounded nearly to the basal levels, but was significantly higher in the CLA-diet group than in the control diet group during the following $2 \mathrm{~h}$ (Figures $4 \mathrm{a}$, $P<0.05-0.001)$. Thus, the same amount of insulin did not reduce serum glucose levels to the same extent in the CLA-containing diet group as it did in the control diet group, clearly indicating a marked insulin resistance status in mice fed the CLA-containing diet, which was supported by fasting serum insulin levels and the calculation of HOMA-insulin resistance as shown in Figure. $4 \mathrm{~b}(P<0.05-0.01)$.

In order to determine whether the insulin resistance seen in mice fed the CLA-containing diet was the result of defects in the insulin signaling, we examined the phosphorylation of Akt, which is a downstream molecule in the insulin-signaling pathway. As shown in Figure 4c, the phosphorylated Akt was much lower in the liver of mice fed the CLA-containing diet than that of control diet group, and the densitometric analysis further confirmed the results (Figure 4d). These results imply that the marked insulin resistance seen in mice fed the CLA-containing diet resulted partially from this signaling defect.

\section{Excess Fatty Acid Accumulation Resulting from Decreased Oxidation and Increased Synthesis}

In order to further examine the sources of excess fatty acids for lipotoxicity and insulin resistance in liver cells, we performed a quantitative RT-PCR assay to evaluate expression of the genes involved in fatty acid oxidation and synthesis. Carnitine palmitoyl transferase- $1 \alpha(\mathrm{CPT}-1 \alpha)$ is an enzyme participating in the mitochondrial translocation of acetyl CoA from the cytosol into the mitochondrial matrix for $\beta$-oxidation, and it is the key enzyme of the carnitine cycle. Acyl-CoA oxidase 1 (ACOX1) is the first rate-limiting enzyme for fatty acid $\beta$-oxidation. PPAR $\alpha$ is a nuclear receptor/transcription factor crucial for regulating lipid metabolism; the lack of PPAR $\alpha$ enhanced hepatic steatosis in PPAR $\alpha$-null mice after feeding an HFC diet. Gene expression of ACOX1, CTP1 $\alpha$ and PPAR $\alpha$ was markedly diminished in mice fed the CLA-containing diet in comparison with those

Figure 3 Coherent anti-stokes Raman scattering (CARS) imaging of lipid droplets in the mouse liver. (a) Liver sections from mice on the control diet showed only few lipid droplet accumulations, whereas liver sections from mice fed the conjugated linoleic acid (CLA)-containing diet showed an accumulation of large, up to $30 \mu \mathrm{m}$ in diameter size, multiple lipid droplets with hepatocytes (b). In this image, it could be seen that some of the lipid in the lipid droplet cavities leaked out due to the microtome slicing that could perturb the lipid inclusions. (c) Representative results of Raman micro-spectral analysis of the fatty acid profile of lipid droplets from the liver of mice fed the CLA-containing diet. $y$ axis represents the signal intensity, and $x$ axis represents Raman shift in per $\mathrm{cm}$. The average spectrum from 20 different lipid droplets is shown. SFA, saturated fatty acid; USFA, unsaturated fatty acid; EFA, esterified fatty acid. 


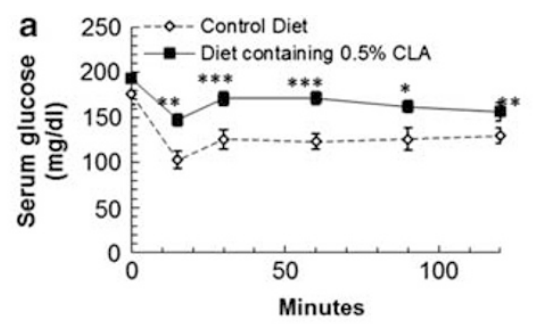

C

Control diet $0.5 \%$ CLA diet
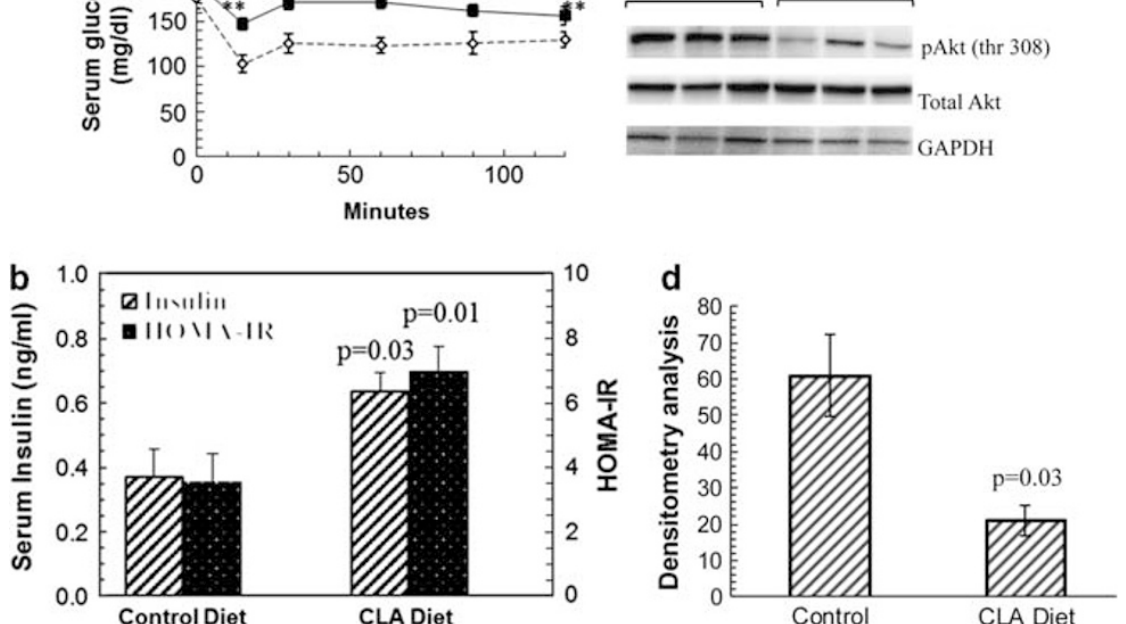

Figure 4 Insulin resistance in mice fed the conjugated linoleic acid (CLA)-containing diet. Insulin resistance was determined by insulin tolerance test (ITT) (a) and calculation of homeostasis model assessment of insulin resistance (HOMA-IR) based on fasting insulin level (mU/I) $\times$ glucose $(\mathrm{mmol} / \mathrm{l}) /$ 22.5. (b) Data were summarized from six mice in each group, and ${ }^{*}, * *$ and ${ }^{* *}$ denote $P<0.05,0.01$ and 0.005 , respectively, at corresponding time points compared with the control diet group. Phosphorylation of Akt at the threonine residue 308 was determined with specific antibodies in the liver of mice fed either control diet or CLA-containing diet $(n=3)$, using total unphosphorylated Akt and GAPDH as loading controls (c). Densitometric analysis of western blot imaging was used to confirm the results (d).

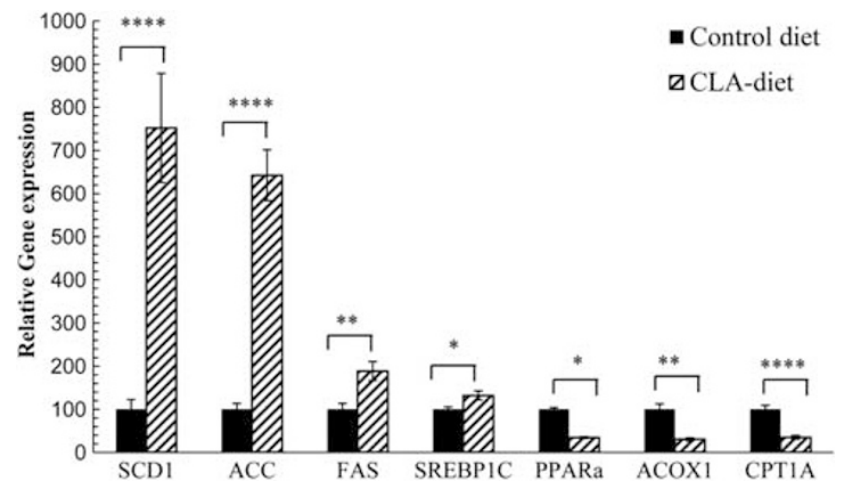

Figure 5 Gene expression of critical enzymes involved in fatty acid synthesis and $\beta$-oxidation. Gene expression of SCD1, ACC, PPAR $\alpha$, SREBP1C, FAS, PPAR $\alpha$, ACOX1 and CPT1A in mouse liver was determined by quantitative RT-PCR using $\beta$-actin as a house-keeping control gene ( $n=6$ in each group). ACC, acetyl CoA carboxylase; ACOX1, acyl-CoA oxidase 1; palmitoyl; CPT- $1 \alpha$, carnitine palmitoyl transferase- $1 \alpha$; FAS, fatty acid synthase; PPAR $\alpha$, peroxisome proliferator-activated receptor- $\alpha$; SCD 1, stearoyl-COA desaturase; SREBP1, sterol regulatory element-binding protein-1. ${ }^{*}, * *, * * * *$ denote $P<0.05,0.01$ and 0.001 compared with the control diet group.

fed the control diet $(P<0.05-0.005)$. In contrast, gene expression of the fatty acid synthesis rate-limiting enzyme, stearoylCoA desaturase-1 (SCD1), a delta-9 fatty acid desaturase that catalyzes the synthesis of $16: 1(n-7)$ and $18: 1(n-9),{ }^{21}$ ACC, FAS and SREBP1 was strikingly upregulated more than 7-fold, 6-fold, nearly 2 -fold and 1.3 -fold in mice fed the diet containing CLA in comparison with those fed the control diet
$(P<0.05-0.001)$ (Figure 5). Thus, it appears that excess accumulation of free fatty acids in hepatocytes, lipid-associated toxicity and insulin resistance may result from accelerated production and suppressed $\beta$-oxidation of fatty acids for energy production in the mitochondria.

\section{DISCUSSION}

In previous studies, we reported that mice fed the CLAcontaining diet developed severe steatosis with increased triglycerides and total lipids in the liver, decreased serum leptin and adiponectin. ${ }^{12}$ We also defined the changes of the fatty acid profile in mouse liver, and found that the n-3-PUFA content was reduced, whereas, the ratio of $\mathrm{n}$-6-PUFA/n-3-PUFA was increased after the feeding the diet containing CLA. These changes were reversible with the concomitant supplementation of flaxseed oil in the diet. ${ }^{12,20}$ Moreover, the body fat mass shrank significantly in mice fed the CLA-containing diet. ${ }^{20}$ Gene expression profile of these mice indicates that there was a dramatic change in lipid and fatty acid metabolism after the feeding the CLA-containing diet for 8 weeks. ${ }^{22}$ In the present study, we characterized the lipotoxicity, fibrosis and insulin resistance associated with hepatic steatosis caused by feeding the diet containing CLA, and confirmed that the fat accumulation led to lipotoxicity and hepatocellular death through oxidant stress, ER stress and apoptosis. These changes further progressed to early fibrosis, and were accompanied with insulin resistance, which may worsen lipotoxicity and promote the progression to steatohepatitis and fibrosis. Thus, we claim here that fatty liver caused by feeding the diet containing CLA represents the 
histopathological features of NASH in humans with both steatohepatitis and marked insulin resistance. This model is at least equivalent, if not better than existing models of NASH, such as those caused by HFC or MCD diets. The further characterization of this model provides a valuable tool in the study of NASH pathophysiology and assessment of possible therapeutic interventions.

In the present study, we confirmed the existence of insulin resistance by ITT and HOMA-IR calculation. In order to understand the mechanism of insulin resistance, we found that there was a significant decrease in the phosphorylation levels of the threonine residue at 308 of the Akt signaling molecule of the insulin receptor. Therefore, the defect in insulin downstream signaling is partially responsible for insulin resistance in this model. We have shown previously that the gene expression of diacylglycerol O-acyltransferase, which is involved in diacylglycerol biosynthesis, was upregulated in the liver of this model. ${ }^{22}$ Liver diacylglycerol content was increased in mice fed the CLA-diet. ${ }^{23}$ Hence, it appears that increased diacylglycerol levels may be associated with the development of insulin resistance by enhancing membrane translocation of protein kinase $\mathrm{C}-\delta$, which blocks the normal phosphorylation of insulin receptor substrate (IRS-1/2) and downstream PI3K and Akt activation. ${ }^{24}$

In the present study, we employed a biophotonic technology, CARS, to image lipid droplets without any fluorescence labeling, and were able to visualize lipid droplets in liver tissues. ${ }^{25}$ Our CARS setup is capable of determining the smallest changes in the amount of neutral lipid by monitoring the formation, mobility and dynamic changes of lipid droplets within living hepatocytes after exposure to VLDL-derived lipolysis products. ${ }^{19}$ CARS imaging was able to visualize multiple lipid droplets in liver sections from mice fed the CLA-containing diet, and the number and size of lipid droplets in these mice were much greater than those from control mice. The Raman spectrum reflects the chemical compound present in the objects. Due to the relative intensity of the 1660 per $\mathrm{cm}$ peak that arises from a $\mathrm{C}=\mathrm{C}$ stretch vibration, which is an indicator for the average degree of unsaturation, the Raman spectra we acquired from visualized lipid droplets suggest that unsaturated fatty acids were present at a low concentration, eg, less than one unsaturated fatty acid bond per molecule. The presence of the 1730 per $\mathrm{cm}$ peak indicates that esterified fatty acids were present in the lipid droplets. In a separate study, we validated specific prediction of fatty acid profile of lipid droplets within hepatic cells by Raman spectroscopy with data from gas chromatographic analysis of fatty acid content. ${ }^{26}$ The data of Raman spectral prediction are consistent with the data by mass spectral analysis of fatty acids. ${ }^{20}$ These findings confirmed the notion that there is a higher ratio of saturated fatty acid over unsaturated fatty acids in lipid droplets. This correlation suggests the reliability of the Raman spectral analysis and its potential use in predicting the severity of fat accumulation and lipotoxicity.
Severe hepatic macrosteatosis in mice fed the diet containing CLA is characterized as the mobilization of lipolysis products, such as fatty acids to hepatocytes from body fat, as evidenced by striking reduction of body fat size and adipocyte cell mass, as well as an increase in liver weight and lipid content. ${ }^{20}$ Owing to significant insulin resistance in the liver and peripheral tissues, such as muscles or adipose tissue, the mobilized lipids were not for energy metabolism, but for accelerated fatty acid synthesis in hepatocytes as evidenced by decreased gene expression of the rate-limiting enzymes (CPT1 and ACOX1) in the $\beta$-oxidation of fatty acids. ${ }^{27}$ Both $C P T$ $1 \alpha$ and ACOX 1 are the target genes of PPAR $\alpha^{27}$ that was downregulated in mice fed the diet containing CLA as seen in this study. In contrast, gene expression of critical enzymes for fatty acid synthesis, such as SCD1, ACC and FAS, as well as lipogenic transcription factor, SREBP1, was profoundly upregulated in the livers of the mice fed the diet containing CLA. The overall consequence is a marked increase of free fatty acids in hepatocytes due to the increased influx of fatty acids, reduced consumption and accelerated production. Numerous studies by us and others have proven that lipotoxicity from oxidized or free fatty acids through oxidant stress ${ }^{19,28}$ and/or ER stress, ${ }^{29}$ both of which lead to cell death through necrosis and/or apoptosis. Feeding the CLA-containing diet also triggered the activation of HSCs for the initiation and progression of hepatic fibrosis, as we saw macrosteatotic features with focal cell death, more SMApositive (activated) HSCs, and pericellular and portal deposition of connective tissue in the histology. ${ }^{16}$ More CD163-positive cells seen in the liver fed the CLA-containing diet indicate the recruitment of Kupffer cells in the mediation of the inflammatory responses. The Kupffer cell recruitment promotes the progression of NASH through the activation of monocyte chemo-attractant protein receptor, CCR2, which are expressed on Kupffer cells and HSCs. ${ }^{30}$ During NASH development, insufficient evidence exists for precisely dissecting whether insulin resistance is an initiator, accelerator or consequence of oxidant stress, ER stress and cell death; however, it appears likely that insulin resistance contributes to a deteriorating loop leading to disordered lipid/fatty acid metabolism $\rightarrow$ steatosis $\rightarrow$ lipotoxicity $\rightarrow$ hepatocellular injury/death $\rightarrow$ worsening lipid/energy metabolic status or crisis. Thus, in this context, it is conceivable that the significant insulin resistance participates in the initiation and progression of steatohepatitis in this model.

Proapoptotic CHOP is a major molecule in the mediation of ER stress. It is activated by AFT4 and AFT6, and triggers ER stress. At the same time, it activates bak leading to cytochrome $c$ release from mitochondria and initiates apoptosis via a mitochondrial pathway. ${ }^{29}$ In turn, ER stress activates c-Jun N-terminal kinases (JNK), which leads to abnormal phosphorylation of IRS-1, and results in disrupted downstream insulin signaling. ${ }^{31}$ We confirmed the enhanced liver ER stress status in the mice fed the CLA-diet by determining $\mathrm{CHOP}$ gene expression. This result delineates 
additional pathways of fatty acid-caused lipotoxicity through oxidant stress and ER stress. It has been well known that oxidant stress causes insulin resistance, ${ }^{32}$ probably partially through ER stress, which needs further investigation in this model.

Autophagy is a lysosome-mediated catabolic process that gives rise to degradation and recycling of all major components of eukaryotic cells. Excess lipid accumulation, lipid-associated oxidant stress and ER stress induce autophagy; and steatotic cells use microlipophagy as a mechanism to break down excess lipid droplets. ${ }^{33-35}$ The engulfment of cytoplasmic materials will form the autophagosomes, which finally merge with lysosomes for further digestion. During this process, LCIII $\beta$ is a regulatory protein, which is present in autophagosomes until they fuse with lysosomes, and thus it has been used as a marker for autophagy. We found that more cells were positive for LCIII $\beta$, indicating an enhanced autophagic process in the liver of mice fed the CLA-diet compared with the control diet, and the data are consistent with other steatotic conditions such as alcoholic toxicity and HCV infection. ${ }^{36,37}$ However, whether enhanced microlipophagy serves as a protective mechanism or a consequence of ER stress remains to be investigated. For such a study, the combination of biophotonic technologies such as deconvolution fluorescence microscopy with CARS will enable to track the subcellular sources of lipid droplet formation and to dissect the relationship of ER stress and autolipophagy.

In conclusion, the findings in the present study verify the similarity of a mouse NASH model to human disease, in terms of lipotoxicity, hepatocellular injury and death, fibrosis and marked insulin resistance. The insulin resistance is partially caused by the defects in the receptor signaling, such as decreased phosphorylation of Akt molecule, as well as oxidant stress and ER stress. This model is particularly useful for further investigation of the critical role of insulin resistance in the initiation and progression of NASH to fibrosis/cirrhosis, as well as investigating the efficacy of insulin sensitizers, such as thiazolidinediones, antioxidants, nutritional supplements or probiotics, etc in the treatment of NASH.

\section{ACKNOWLEDGMENTS}

We thank Dr Rajen Ramsamooj in the Department of Pathology and Laboratory Medicine, UC Davis Medical Center for technical assistance in the process of tissue sections for H\&E and Trichrome staining. The study was supported by the United States Department of Agriculture, Agricultural Research Service, through Parent project (5306-51530-017-00D) to the Immunity and Disease Prevention Unit at Western Human Nutrition Research Center; and by the NIH Grant to JW (DK069939).

Special notes: Reference to a company or product name does not imply an approval or recommendation of the product by the U.S. Department of Agriculture to the exclusion of others that may be suitable. USDA is an equal opportunity employer and provider.

\section{DISCLOSURE/CONFLICT OF INTEREST}

The authors declare no conflict of interest.
1. Charlton MR, Burns JM, Pedersen RA, et al. Frequency and outcomes of liver transplantation for nonalcoholic steatohepatitis in the United States. Gastroenterology 2011;141:1249-1253.

2. Stein LL, Dong MH, Loomba R. Insulin sensitizers in nonalcoholic fatty liver disease and steatohepatitis: Current status. Adv Ther 2009;26:893-907.

3. Sanyal AJ, Chalasani N, Kowdley KV, et al. Pioglitazone, vitamin E, or placebo for nonalcoholic steatohepatitis. New Engl J Med 2010;362: 1675-1685.

4. Chalasani N, Younossi Z, Lavine JE, et al. The diagnosis and management of non-alcoholic fatty liver disease: Practice Guideline by the American Association for the Study of Liver Diseases, American College of Gastroenterology, and the American Gastroenterological Association. Hepatology 2012;55:2005-2023.

5. Musso G, Gambino R, Cassader M, et al. A meta-analysis of randomized trials for the treatment of nonalcoholic fatty liver disease. Hepatology 2010;52:79-104.

6. Zein CO, Yerian LM, Gogate $P$, et al. Pentoxifylline improves nonalcoholic steatohepatitis: a randomized placebo-controlled trial. Hepatology 2011;54:1610-1619.

7. Gibson LC, Hastings SF, McPhee I, et al. The inhibitory profile of Ibudilast against the human phosphodiesterase enzyme family. Eur J Pharmacol 2006;538:39-42.

8. Wu J. Coumarin: an alternative candidate for the treatment of nonalcoholic steatohepatitis? Br J Nutr 2013;109:1542-1543.

9. Schattenberg JM, Galle PR. Animal models of non-alcoholic steatohepatitis: of mice and man. Dig Dis 2010;28:247-254.

10. Vinciguerra $M$, Veyrat-Durebex $C$, Moukil MA, et al. PTEN downregulation by unsaturated fatty acids triggers hepatic steatosis via an NF-kappaBp65/mTOR-dependent mechanism. Gastroenterology 2008;134:268-280

11. Kawai D, Takaki A, Nakatsuka A, et al. Hydrogen-rich water prevents progression of nonalcoholic steatohepatitis and accompanying hepatocarcinogenesis in mice. Hepatology 2012;56:912-921.

12. Vemuri M, Kelley DS, Mackey BE, et al. Docosahexaenoic acid (DHA) but not eicosapentaenoic acid (EPA) prevents trans-10, cis-12 conjugated linoleic acid (CLA)-induced insulin resistance in mice. Metab Synd Related Dis 2007;5:315-322.

13. Kelley DS, Vemuri $M$, Adkins $Y$, et al. Flaxseed oil prevents trans-10, cis-12-conjugated linoleic acid-induced insulin resistance in mice. $\mathrm{Br} \mathrm{J}$ Nutr 2009;101:701-708.

14. Zhu J, Wu J, Frizell E, et al. Rapamycin inhibits hepatic stellate cell proliferation in vitro and limits fibrogenesis in an in vivo model of liver fibrosis. Gastroenterology 1999;117:1198-1204.

15. Wu J, Liu L, Yen RD, et al. Liposome-mediated extracellular superoxide dismutase gene delivery protects against acute liver injury in mice. Hepatology 2004;40:195-204.

16. Zhan SS, Jiang JX, Wu J, et al. Phagocytosis of apoptotic bodies by hepatic stellate cells induces NADPH oxidase and is associated with liver fibrosis in vivo. Hepatology 2006;43:435-443.

17. Li F, Song Z, Li Q, et al. Molecular imaging of hepatic stellate cell activity by visualization of hepatic integrin alphavbeta3 expression with SPECT in rat. Hepatology 2011;54:1020-1030.

18. Lingala $S, C u i \quad Y Y, C$ en $X$, et al. Immunohistochemical staining of cancer stem cell markers in hepatocellular carcinoma. Exp Mol Pathol 2010;89:27-35.

19. Schie IW, Wu J, Weeks T, et al. Label-free imaging and analysis of the effects of lipolysis products on primary hepatocytes. J Biophotonics 2011:4:425-434.

20. Fedor DM, Adkins Y, Newman JW, et al. The effect of docosahexaenoic acid on t10, c12-conjugated linoleic acid-induced changes in fatty acid composition of mouse liver, adipose, and muscle. Metab Synd Related Dis 2013;11:63-70.

21. Narce M, Bellenger J, Rialland $M$, et al. Recent advances on stearoylCoA desaturase regulation in fatty liver diseases. Curr Drug Metab 2012;13:1454-1463.

22. Rasooly R, Kelley DS, Greg J, et al. Dietary trans 10 , cis 12 -conjugated linoleic acid reduces the expression of fatty acid oxidation and drug detoxification enzymes in mouse liver. Br J Nutr 2007;97:58-66.

23. Stout MB, Liu LF, Belury MA. Hepatic steatosis by dietary-conjugated linoleic acid is accompanied by accumulation of diacylglycerol and increased membrane-associated protein kinase $\mathrm{C}$ epsilon in mice. Mol Nutr Food Res 2011;55:1010-1017. 
24. Jornayvaz FR, Birkenfeld $A L$, Jurczak $M J$, et al. Hepatic insulin resistance in mice with hepatic overexpression of diacylglycerol acyltransferase 2. Proc Natl Acad Sci USA 2011;108:5748-5752.

25. Chan JW, Motton D, Rutledge JC, et al. Raman spectroscopic analysis of biochemical changes in individual triglyceride-rich lipoproteins in the pre- and postprandial state. Anal Chem 2005;77:5870-5876.

26. Schie IW, Nolte L, Pedersen TL, et al. Determining fatty acid ratios in single cellular lipid droplets using Raman spectroscopy and CARS microscopy. Analyst 2013;138:6662-6670.

27. Vluggens A, Andreoletti P, Viswakarma N, et al. Reversal of mouse Acyl-CoA oxidase 1 (ACOX1) null phenotype by human ACOX1b isoform. Lab Invest 2010;90:696-708.

28. Fuchs M, Sanyal AJ. Lipotoxicity in NASH. J Hepatol 2012;56:291-293.

29. Malhi H, Kaufman RJ. Endoplasmic reticulum stress in liver disease. J Hepatol 2011;54:795-809.

30. Miura K, Yang L, van Rooijen N, et al. Hepatic recruitment of macrophages promotes nonalcoholic steatohepatitis through CCR2. Am J Physiol Gastrointestinal Liver Physiol 2012;302:G1310-G1321.
31. Hirosumi J, Tuncman G, Chang L, et al. A central role for JNK in obesity and insulin resistance. Nature 2002;420:333-336.

32. Seo YS, Kim JH, Jo NY, et al. PPAR agonists treatment is effective in a nonalcoholic fatty liver disease animal model by modulating fatty-acid metabolic enzymes. J Gastroenterol Hepatol 2008;23:102-109.

33. Bechmann LP, Hannivoort RA, Gerken G, et al. The interaction of hepatic lipid and glucose metabolism in liver diseases. J Hepatol 2012;56:952-964.

34. Liu K, Czaja MJ. Regulation of lipid stores and metabolism by lipophagy. Cell Death Different 2013;20:3-11.

35. Singh R, Kaushik $S$, Wang $Y$, et al. Autophagy regulates lipid metabolism. Nature 2009;458:1131-1135.

36. Ding WX, Li M, Chen X, et al. Autophagy reduces acute ethanolinduced hepatotoxicity and steatosis in mice. Gastroenterology 2010;139:1740-1752.

37. Vescovo $T$, Romagnoli $A$, Perdomo $A B$, et al. Autophagy protects cells from HCV-induced defects in lipid metabolism. Gastroenterology 2012;142:e643. 